\title{
Ventilation Efficacy of Video-Laryngoscopes Equipped With a Ventilation Feature
}

\author{
Jun Oto MD PhD, Christopher T Chenelle, Zhenbo Su MD PhD, Mary Q Sun, \\ Yandong Jiang MD PhD, and Robert M Kacmarek PhD RRT FAARC
}

\begin{abstract}
INTRODUCTION: Achieving effective ventilation is challenging for anesthesia care providers and emergency medical personnel, as difficult mask ventilation and difficult intubation frequently occur. The aim of this study was to determine whether video-laryngoscopes equipped with a ventilation feature can produce effective ventilation. METHODS: An intubation mannequin with its trachea connected to a model lung with compliance 50 (normal compliance: C50) and $20 \mathrm{~mL} / \mathrm{cm}$ $\mathrm{H}_{2} \mathrm{O}$ (low compliance: $\mathrm{C20}$ ) was used. Ventilation was established via a ventilation catheter (inner diameter $3.5 \mathrm{~mm}, 50 \mathrm{~cm}$ length) extending to the tip of the video-laryngoscope blade. Three different views of the vocal cords (grade 1, vocal cords fully visualized; grade 2, partial vocal cord visualization; grade 3, only epiglottis visualized) were tested. Ventilation was provided by jet ventilator (Jet). The Jet was operated at 10, 15, and 20 psi (Jet10, Jet15, and Jet20). Effective tidal volume $\left(V_{T}\right)$ was defined as a $V_{T}$ greater than anatomical dead space $(150 \mathrm{~mL})$. RESULTS: In C50, Jet15 and Jet20 generated effective $V_{T}$ in all vocal cord views (for Jet15: grade 1, $663 \pm 33 \mathrm{~mL}$; grade 2, $363 \pm 25 \mathrm{~mL}$; and grade 3, $198 \pm 9 \mathrm{~mL}$; for Jet20: grade 1, 1,005 $\pm 114 \mathrm{~mL}$; grade 2, $484 \pm 38 \mathrm{~mL}$; grade 3, $268 \pm 8 \mathrm{~mL}$, respectively). In C20, Jet15 and Jet20 generated effective $V_{T}$ in grades 1 and 2 (Jet15: grade 1, $288 \pm 8 \mathrm{~mL}$; grade 2, $160 \pm 20 \mathrm{~mL}$; grade 3, $81 \pm 7 \mathrm{~mL}$; Jet20: grade 1, $421 \pm 20 \mathrm{~mL}$; grade $2,222 \pm 16 \mathrm{~mL}$; grade 3, $111 \pm 8 \mathrm{~mL}$, respectively). Jet10 achieved effective $V_{T}$ in grade 1 and 2 (grade 1, $354 \pm 6 \mathrm{~mL}$; grade $2,223 \pm 37 \mathrm{~mL}$, respectively) in C50 and grade $1(163 \pm 12 \mathrm{~mL})$ in C20. CONCLUSIONS: Video-laryngoscopes equipped with a ventilation feature provided effective $V_{T}$ in simulated clinical scenarios. Further clinical study is required to validate these findings. Key words: video-laryngoscope; ventilation catheter; endotracheal intubation; difficult airway; emergency ventilation; jet ventilation. [Respir Care 2014;59(11):1636-1642. (C) 2014 Daedalus Enterprises]
\end{abstract}

\section{Introduction}

Difficult mask ventilation and intubation are major causes of morbidity and mortality in emergency and anesthesia care. Difficult tracheal intubation occurs in 1-4\% of general surgical patients ${ }^{1}$ and $4-11 \%$ in the emergency department. ${ }^{2,3}$ During field resuscitation, intubation is even more challenging. ${ }^{4,5}$ Intubation performed during chest compression and/or cervical stabilization in trauma pa-

Dr Oto, Mr Chenelle, and Dr Kacmarek are affiliated with Respiratory Care Services, Massachusetts General Hospital; and Dr Su, Ms Sun, and Dr Jiang are affiliated with Anesthesia, Critical Care, and Pain Medicine, Massachusetts General Hospital, Boston, Massachusetts.

This research was supported by departmental funding. tients creates additional difficulty. 6,7 The major cause of intubation-related brain damage or death is lack of adequate oxygenation and ventilation. The American Society of Anesthesiologists closed claim analysis of anesthesia complications indicates that respiratory-related events were responsible for $50 \%$ or more of the claims for death or

\footnotetext{
Dr Kacmarek received a research grant from Covidien and Hollister and has received an honorarium for lecturing from Maquet. All other authors declare no conflicts of interest.

Correspondence: Robert M Kacmarek PhD RRT, FAARC, Respiratory Care Services, Massachusetts General Hospital, 55 Fruit Street, Boston, MA 02114. E-mail: rkacmarek@partners.org.
}

DOI: $10.4187 /$ respcare. 03169 
permanent brain damage. ${ }^{8}$ In the respiratory events category, the most frequently seen events were difficult intubation (23\%) and inadequate ventilation/oxygenation (22\%). ${ }^{8}$ In addition, patients at risk for desaturation such as critically ill patients, obese patients, pregnant patients, and others show rapidly developing hypoxemia during intubation $^{9,10}$ Therefore, it is important to minimize the apneic period, especially in patients at risk for desaturation, and to achieve effective ventilation during intubation, particularly when intubation is difficult or impossible. However, achieving these goals remains challenging for anesthesia care providers and emergency medical personnel.

Recently, video-laryngoscopes, enabling the operator to achieve better and quicker views than with direct laryngoscopes, have become available for use in operating rooms, intensive care units, and emergency departments. ${ }^{11}$ Studies also demonstrated that video-laryngoscopes provide a higher intubation success rate than conventional laryngoscopes. ${ }^{11-13}$ However, it is unclear whether the use of video-laryngoscopes shortens the time to achieve successful intubation. ${ }^{14,15}$ Because the video-laryngoscope itself cannot provide adequate oxygenation and ventilation, better visualization of the vocal cords in the absence of quicker intubation may produce a false sense of security and does not translate into a reduction of hypoxia during intubation. In contrast, if ventilation is provided during intubation, it potentially avoids or minimizes hypoxia due to a lack of ventilation and affords the practitioner additional time to consider alternative airway management.

In this study, we evaluated a prototype of a videolaryngoscope equipped with the ventilation feature (VLsVent) using a jet ventilator (Jet). We hypothesized that the VLs-Vent would generate effective ventilation during endotracheal intubation. This hypothesis was tested on an intubation mannequin and lung model simulating respiratory system compliances potentially encountered in clinical scenarios.

\section{Methods}

\section{Study Setup (Lung Model and Mannequin)}

The study was conducted on an intubation mannequin (Laerdal Airway Management Trainer, Laerdal Medical, Stavanger, Norway) with its trachea connected to a lung model (Dual Adult TTL training/test lung, model 1600, Michigan Instruments, Grand Rapids, Michigan) (Fig. 1). The lung model had a functional residual capacity of $1,020 \mathrm{~mL}$, and was connected to the distal end of an artificial trachea with anatomical dead space of approximately $150 \mathrm{~mL}$. The lung model compliance was $50 \mathrm{~mL} / \mathrm{cm} \mathrm{H}_{2} \mathrm{O}$

\section{QUICK LOOK}

\section{Current knowledge}

During difficult tracheal intubation, oxygenation and ventilation can be compromised. A number of methods have been devised to maintain gas exchange during intubation.

\section{What this paper contributes to our knowledge}

In a bench model, the use of high frequency jet ventilation with a modified video-laryngoscope allowed adequate tidal volume delivery with normal and low lung compliance with visualization of the vocal cords. When the vocal cords could not be seen and model lung compliance was low, ventilation was inadequate.

(normal compliance: $\mathrm{C} 50$ ) and $20 \mathrm{~mL} / \mathrm{cm} \mathrm{H}_{2} \mathrm{O}$ (low compliance: C20). Airway resistance was set at $5 \mathrm{~cm} \mathrm{H}_{2} \mathrm{O} / \mathrm{L} / \mathrm{s}$. A flow/pressure sensor (NICO cardiopulmonary management system, model 7300, Philips Respironics, Murrysville, Pennsylvania) was placed between the distal end of the mannequin trachea and the model lung (Fig. 1). The expiratory tidal volume $\left(\mathrm{V}_{\mathrm{T}}\right)$, airway pressure, and gas flow were automatically recorded at a sampling rate of $100 \mathrm{~Hz}$. To evaluate for gastric distention, the mannequin esophagus was connected to a PEEP valve, set at $20 \mathrm{~cm}$ $\mathrm{H}_{2} \mathrm{O}$, and a balloon (Fig. 1). The PEEP valve was unidirectional, only allowing air to enter the balloon. Before collecting data, the PEEP valve was tested ensuring that the balloon was inflated when positive-pressure ventilation was applied with a driving pressure greater than $20 \mathrm{~cm}$ $\mathrm{H}_{2} \mathrm{O}$.

\section{Video-Laryngoscope Equipped With Ventilation Feature (VLs-Vent)}

Ventilation was established by mounting a ventilation catheter extending to the tip of the blade of the videolaryngoscope positioned proximal to the vocal cords (Fig. 1). The ventilation catheter was a $19 \mathrm{Fr}$ airway exchanger catheter (Cook Critical Care, Bloomington, Indiana) adjusted to $50 \mathrm{~cm}$ length (Fig. 1). Two different types of video-laryngoscopes, VividTrac (Vivid Medical, Palo Alto, California) and C-MAC (DL, Heine, Dover, New Hampshire), were tested. VividTrac has an angled blade, and an endotracheal tube was preloaded into the tube channel. The ventilation catheter was inserted into the endotracheal tube from the proximal end of the endotracheal tube, and the distal tip of the ventilation catheter was advanced $1 \mathrm{~cm}$ ahead of the tip of the endotracheal tube (Fig. 1). C-MAC has a standard Macintosh blade (size 3) 
A
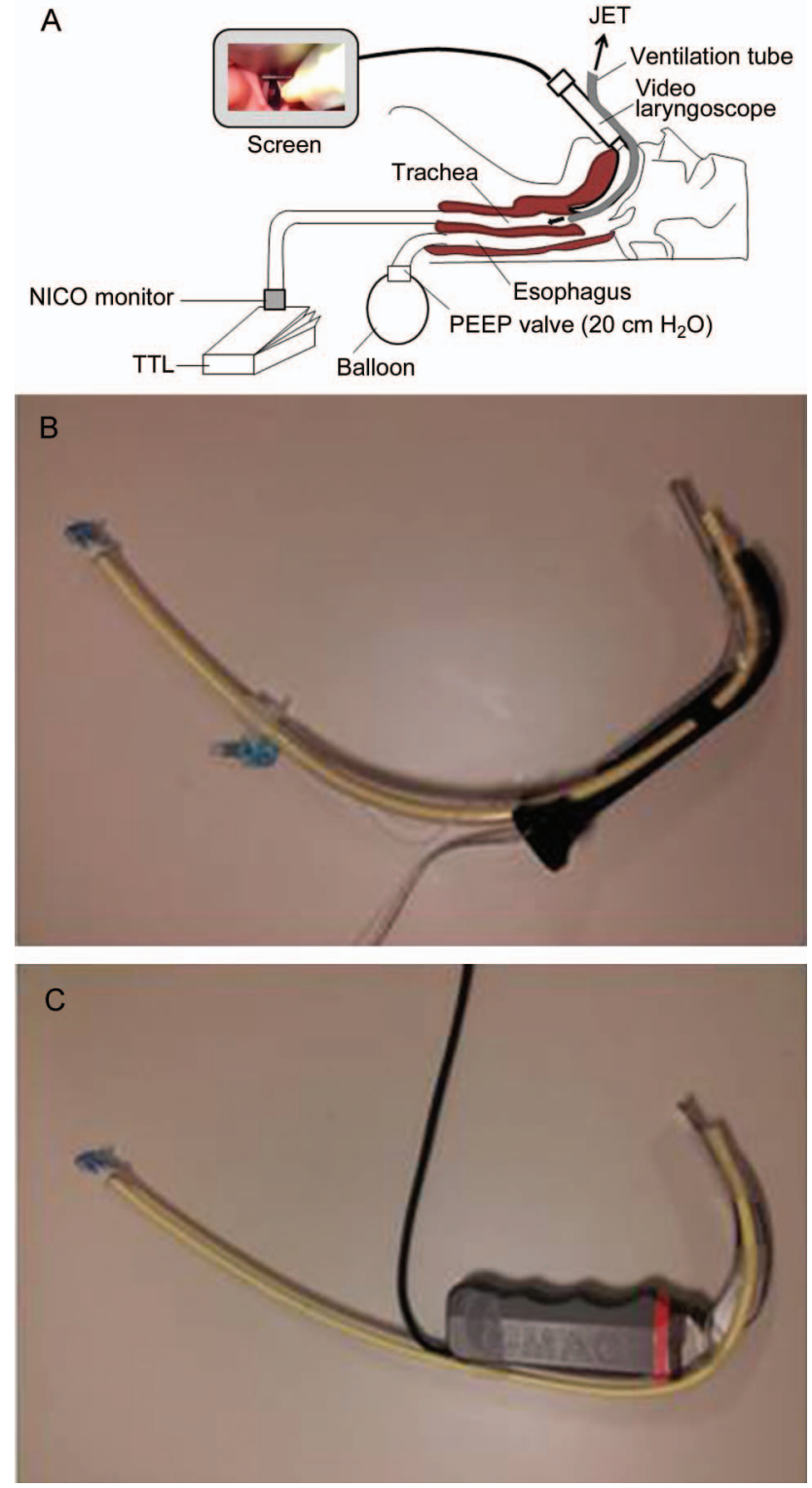

Fig. 1. Experimental setup and video-laryngoscope equipped with a ventilation feature. $A$ : an intubation mannequin with its trachea connected to a model lung. The mannequin's esophagus was connected to a $20 \mathrm{~cm} \mathrm{H}_{2} \mathrm{O}$ PEEP valve and balloon. Ventilation catheter was connected to jet ventilator. $\mathrm{NICO}=$ noninvasive cardiac output monitor; TTL = lung model; JET = jet ventilator. B: ventilation catheter with VividTrac; C: ventilation catheter with C-MAC. The ventilation catheter was inserted into the endotracheal tube when VividTrac was used. The curvature of the endotracheal tube allowed the tip of the ventilation catheter to be aligned with the blade without any mounting. The ventilation catheter was taped to the C-MAC blade to direct the tip of the catheter in alignment with the blade.

and includes a camera. The ventilation catheter was attached to the video-laryngoscope parallel to the angle of its blade (Fig. 1). A jet ventilator (Jet, model 00-325, Anesthesia Associates, San Marcos, CA) was used to pro- vide ventilation. The ventilation catheter was connected directly to the Jet. The Jet was operated at 10,15 , and 20 psi $(68.9,103.4$, and $137.9 \mathrm{kPa})$ (Jet10, Jet15, and Jet20) at a breathing frequency of 15 breaths/min and inspiratoryexpiratory (I:E) ratios of $1: 3$ and $1: 1$ achieved by the operator guided by a timer. Ventilation was performed with 3 different views of the vocal cords (grade 1, fully visible; grade 2, partially visible; and grade 3 , not visible, with epiglottis only visualized) (Fig. 2). ${ }^{16}$

\section{Data Collection and Analysis}

Data from each experimental setting were continuously collected using the Nico Analysis Plus data management system. Establishment of a steady state was generally achieved after 2 or 3 breaths. Data were then collected, analyzed, and averaged from the next 10 consecutive breaths at each experimental setting. Data are presented as the mean $\pm \mathrm{SD}$. Effective $\mathrm{V}_{\mathrm{T}}$ was considered a $\mathrm{V}_{\mathrm{T}}$ greater than anatomical dead space $(150 \mathrm{~mL})$. Continuous data from the lung simulator were compared using the Wilcoxon test to assess the impact of I-E ratio and lung mechanics on $\mathrm{V}_{\mathrm{T}}$. The Friedman test followed by Bonferroni correction for multiple comparisons was used for comparisons between driving pressure or different vocal cord views. Statistical analysis was performed with a statistical software package (PASW Statistic 18; SPSS, Chicago, Illinois). $P<.05$ was considered statistically significant. $\mathrm{V}_{\mathrm{T}}$ differences are only reported if they are both statistically significant $(P<.05)$ and clinically important $(>10 \%$ difference and differences of $>50 \mathrm{~mL}$ ).

\section{Results}

\section{Effects of Driving Pressure and View of Vocal Cords on $V_{T}$ and Airway Pressure}

As vocal cords were more visible and/or driving pressure increased, $\mathrm{V}_{\mathrm{T}}$ increased $(P<.001) . \mathrm{V}_{\mathrm{T}}$ was larger in $\mathrm{C} 50$ than in $\mathrm{C} 20(P<.001)$. In $\mathrm{C} 50$, Jet15 and Jet20 generated effective $\mathrm{V}_{\mathrm{T}}$ in all vocal cord views (Fig. 3). In C20, Jet15 and Jet20 generated effective $\mathrm{V}_{\mathrm{T}}$ in grade 1 and grade 2 but not in grade 3 (Fig. 3). Jet10 achieved effective $\mathrm{V}_{\mathrm{T}}$ in grade 1 and grade 2 in C50 and grade 1 in C20.

Figure 4 shows the peak airway pressure associated with each driving pressure and view of the vocal cords. As vocal cords were more visible and/or driving pressure increased, mean peak airway pressure increased $(P<.001)$. Mean peak airway pressure did not differ significantly between C50 and C20. No gastric distention was observed with any driving pressure or view of the vocal cord. 


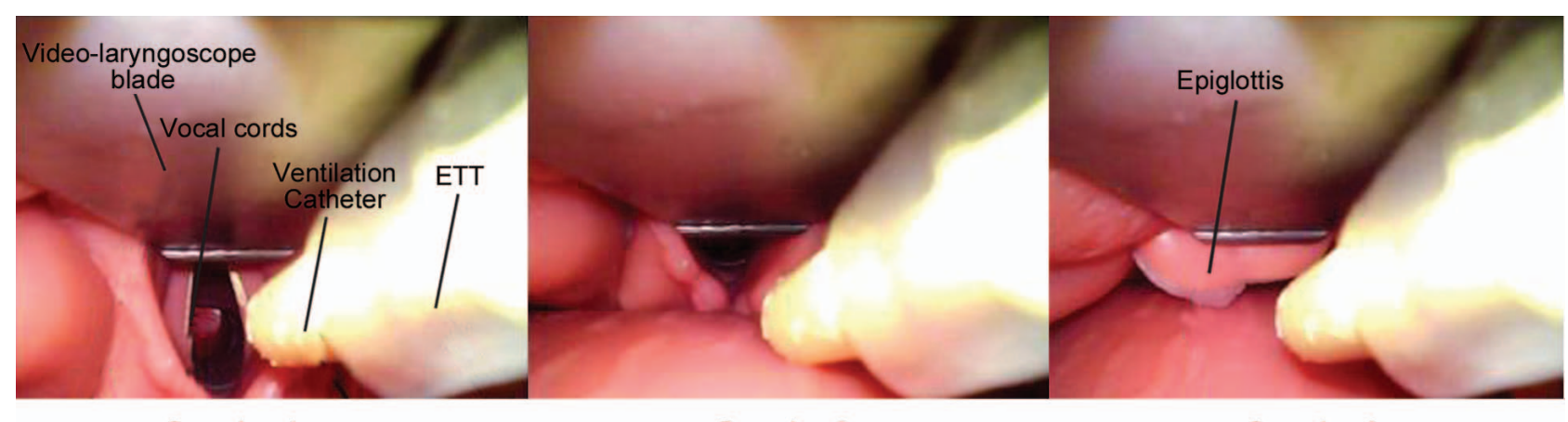

Grade 1

Grade 2

Grade 3

Fig. 2. Three views of the vocal cords taken with the VividTrac. Grade 1: vocal cords fully visible. Photo includes video-laryngoscope blade, vocal cords, endotracheal tube (ETT), and ventilation catheter. Grade 2: vocal cords partially visible; the center of screen was focused on the inlet of esophagus. Grade 3: vocal cords not visible; only the epiglottis is visualized.
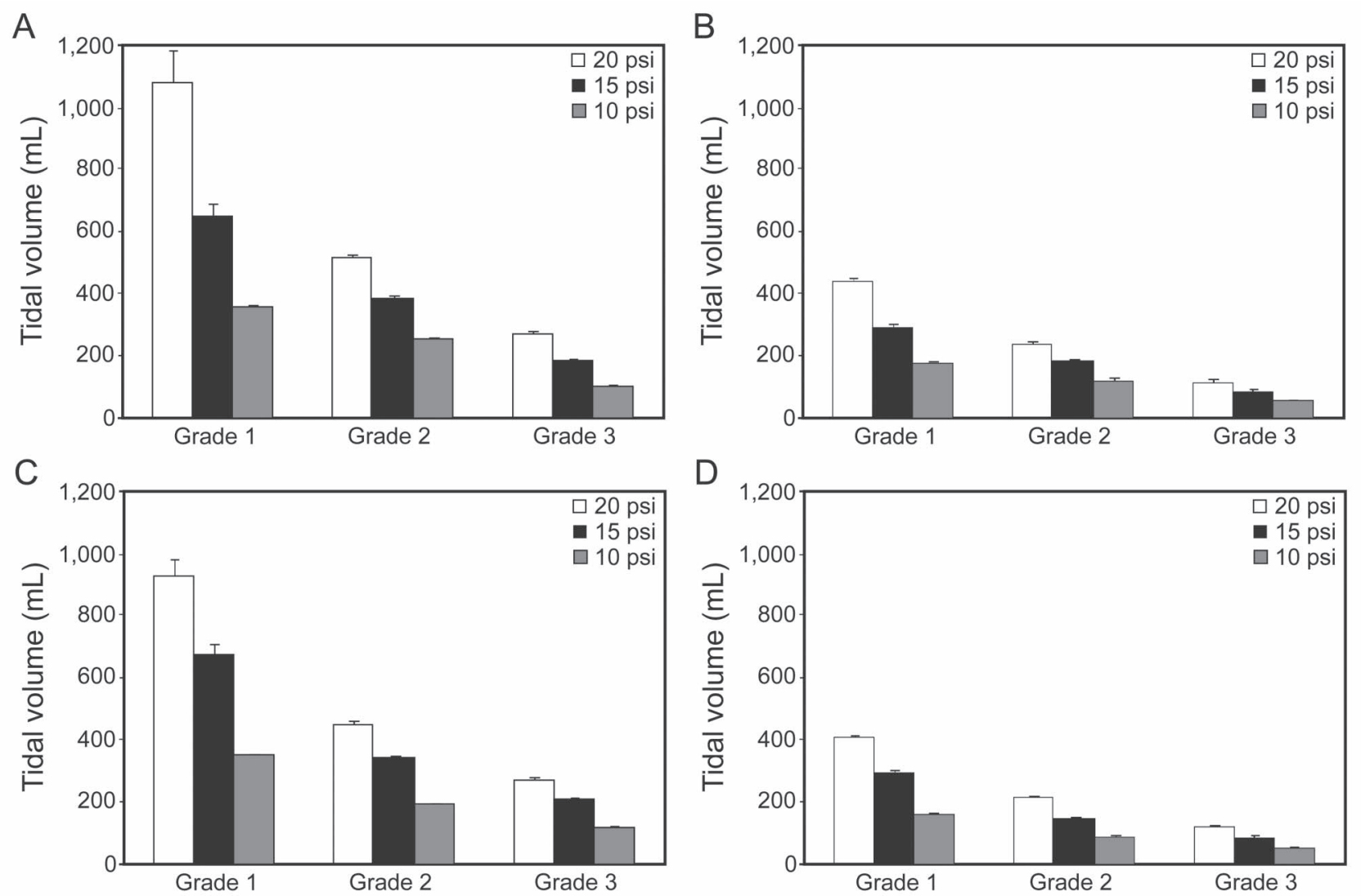

Fig. 3. Tidal volume at each driving pressure. A: VividTrac with normal compliance (50). B: VividTrac with low compliance (20). C: C-MAC with normal compliance (50). D: C-MAC with low compliance (20). Data are shown as mean \pm SD.

Effects of Ratios of Inspiration to Expiration Time on $\mathbf{V}_{\mathbf{T}}$

There were no significant differences in $\mathrm{V}_{\mathrm{T}}$ between I:E 1:3 and 1:1 in C50 (I:E 1:3 vs 1:1: grade 1, $639 \mathrm{~mL}$ vs $710 \mathrm{~mL}$; grade 2, $353 \mathrm{~mL}$ vs $360 \mathrm{~mL}$; grade $3,190 \mathrm{~mL}$ vs $194 \mathrm{~mL} ;<10 \%$ differences) and in C20 (I:E 1:3 vs 1:1: grade 1, $286 \mathrm{~mL}$ vs $296 \mathrm{~mL}$; grade $2,155 \mathrm{~mL}$ vs $164 \mathrm{~mL}$; grade 3, $76 \mathrm{~mL}$ vs $86 \mathrm{~mL} ;<10 \%$ differences). 
A

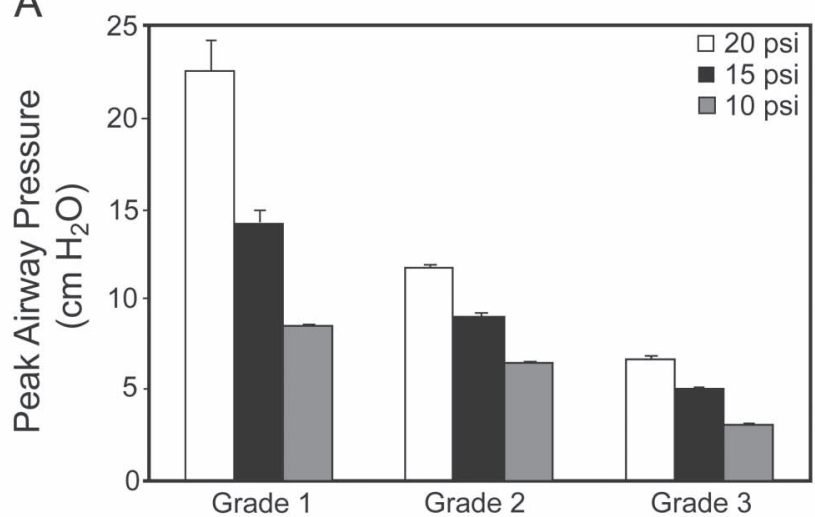

C

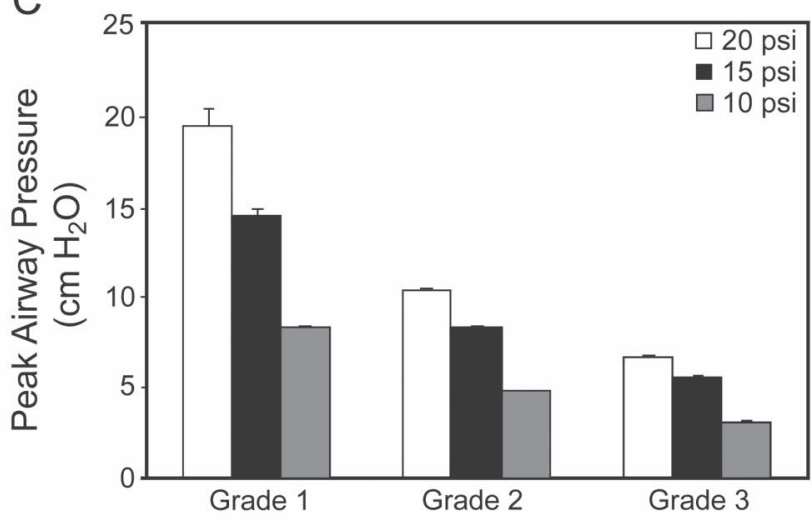

B

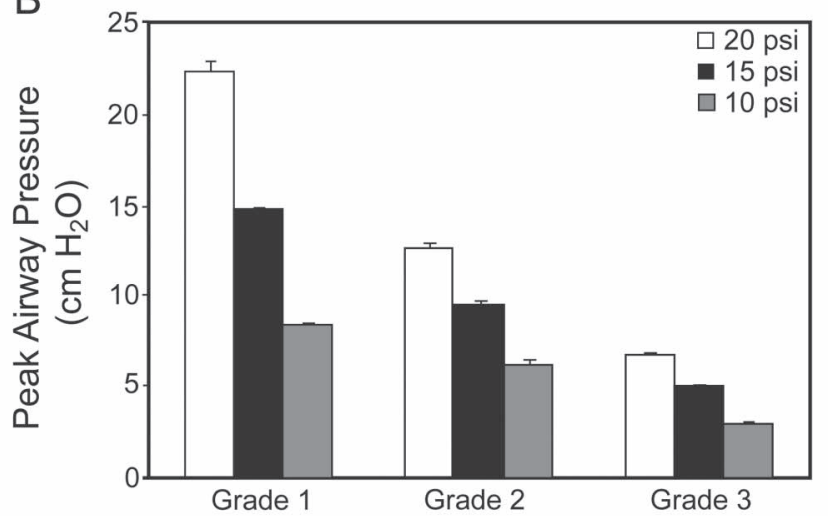

D

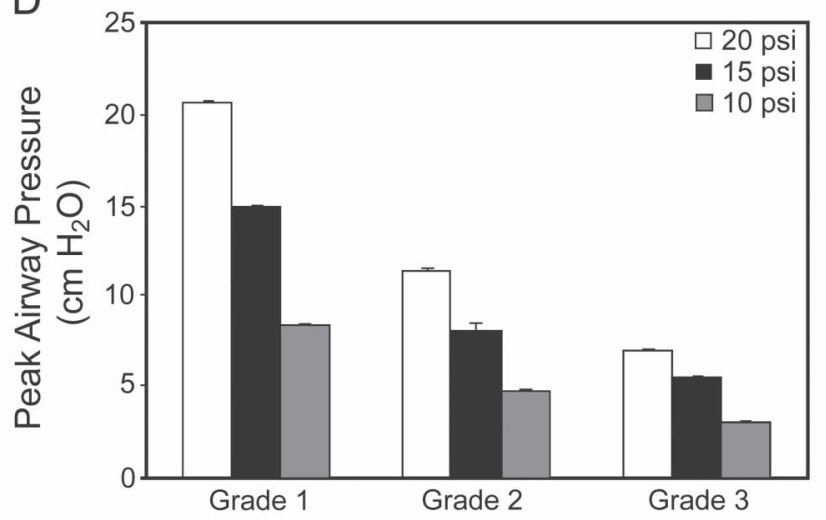

Fig. 4. Peak airway pressure at each driving pressure. A: VividTrac with normal compliance (50). B: VividTrac with low compliance (20). C: C-MAC with normal compliance (50). D: C-MAC with low compliance (20). Data are shown as mean \pm SD.

\section{Effect of Different Types of Video-Laryngoscope on $\mathbf{V}_{T}$}

There was no significant difference in $\mathrm{V}_{\mathrm{T}}$ between the 2 types of video-laryngoscope in C50 (VividTrac vs C-MAC: grade 1, $698 \mathrm{~mL}$ vs $651 \mathrm{~mL}$; grade 2, $389 \mathrm{~mL}$ vs $325 \mathrm{~mL}$; grade 3, $190 \mathrm{~mL}$ vs $195 \mathrm{~mL} ;<10 \%$ differences) and in C20 (VividTrac vs C-MAC: grade 1, $300 \mathrm{~mL}$ vs $281 \mathrm{~mL}$; grade 2, $177 \mathrm{~mL}$ vs $142 \mathrm{~mL}$; grade $3,81 \mathrm{~mL}$ vs $80 \mathrm{~mL}$; $<10 \%$ differences or $<50 \mathrm{~mL}$ differences).

\section{Discussion}

The main findings of this study are as follows: (1) Jet15 and Jet2 0 can produce effective $\mathrm{V}_{\mathrm{T}}$ with normal compliance in all views of vocal cords, (2) Jet15 and Jet20 can produce effective $\mathrm{V}_{\mathrm{T}}$ with low compliance in grade 1 and grade 2 but not in grade 3, (3) Jet10 achieved effective $\mathrm{V}_{\mathrm{T}}$ in grade 1 and grade 2 with normal compliance and grade 1 in low compliance, and (4) there were no differences in $\mathrm{V}_{\mathrm{T}}$ between the 2 video-laryngoscopes. These preliminary data would indicate that a video-laryngoscope equipped with a ventilation feature can produce effective ventilation during intubation. To the best of our knowledge, this is the first study to determine the efficacy of a prototype videolaryngoscope equipped with a ventilation feature.

In this study, the VLs-Vent achieved effective $\mathrm{V}_{\mathrm{T}}$ even when the vocal cords were not visualized. One possible advantage of using the VLs-Vent is that upper airway patency is maintained by lifting the soft tissues directly and the location of the ventilation catheter can be clearly identified during intubation.

The effectiveness of ventilation generated by this device was strongly affected by the grade of vocal cord view and driving pressure. Compared with grade $1, \mathrm{~V}_{\mathrm{T}}$ dramatically decreased in grade 2 and in grade 3 . This may have been partially due to the mouth and upper airway being widely open, offering little resistance to gas leakage from the mouth. Our results are consistent with previous studies showing that, during supraglottic jet ventilation, incorrectly positioning the tip of the jet nozzle dramatically decreases effectiveness of ventilation compared with the nozzle positioned above the vocal cord opening. ${ }^{17,18}$ Jet20 generated effective $V_{T}$ in both normal and low compliance, but caused overinflation in normal compliance with grade 1 view $\left(\mathrm{V}_{\mathrm{T}}>1,000 \mathrm{~mL}\right)$. In contrast, Jet10 generated effective $\mathrm{V}_{\mathrm{T}}$ in normal compliance model as long as the vocal 
cords were partially or fully visible. In previous reports, supraglottic Jet ventilation with an endotracheal tube, combined with a $2.0 \mathrm{~mm}$ inner diameter ventilation tube and using 15 psi driving pressure, maintained adequate oxygenation and ventilation during tracheal intubation. ${ }^{17,18}$ To generate an appropriate $\mathrm{V}_{\mathrm{T}}$, driving pressure must be adjusted based on ventilation catheter location and patient's lung compliance. Considering that a grade 1 and grade 2 view can be obtained in $89-100 \%$ of cases with video-laryngoscopes, ${ }^{14,19,20}$ we expect that our system may be useful and effective for ventilation during most intubations.

Percutaneous transtracheal jet ventilation (PTJV) has been recommended by the American Association of Anesthesiologists and Difficult Airway Society for management of the difficult airway during cannot intubate/cannot ventilate situations. ${ }^{21,22}$ However, a major concern with the use of jet ventilation via percutaneous transtracheal catheter is barotrauma or tissue damage. It has been reported that the incidence of barotrauma during PTJV is as high as $10 \% .^{23}$ These complications were mainly associated with incorrect insertion of the ventilation catheter. With our system, the operator can visualize the tip of the ventilation catheter during ventilation. Therefore, this system in combination with jet ventilation can provide ventilation as effective as PTJV, but it also prevents the damage caused by incorrect placement of the tip of the catheter. Another cause of injury during PTJV is that placement of the tip of the catheter is correct, but ventilation is provided when upper airway obstruction is complete. ${ }^{19}$ In such a case, intra-alveolar pressure can be as high as the driving pressure of jet ventilation. Therefore, catastrophic events may occur including pneumothorax ${ }^{23,24}$ and cardiac arrest $\mathrm{t}^{25}$ due to unrecognized upper airway obstruction during PTJV. In this new system, ventilation is performed via a video-laryngoscopy inserted into the pharyngeal cavity ensuring an open pathway. Therefore, the likelihood of barotrauma caused by unrecognized complete upper away obstruction is eliminated. In previous reports during supraglottic jet ventilation, driving pressure of 14.5-50.8 psi did not show any significant damage to pharyngeal tissue such as barotrauma or subcutaneous emphysema. ${ }^{18,26}$

Another concern with this system is the development of gastric insufflation. In order to determine whether any significant gastric insufflation occurs when ventilation is provided with this system, we simulated lower esophageal sphincter opening pressure by placing a $20 \mathrm{~cm} \mathrm{H}_{2} \mathrm{O}$ PEEP valve on the esophagus (Fig. 1). Although airway pressure reached $22 \mathrm{~cm} \mathrm{H}_{2} \mathrm{O}$ with 20 psi driving pressure, significant gastric distention was not observed during ventilation. Because the opening pressure of the lower esophageal sphincter has been estimated to be approximately $20-25 \mathrm{~cm}$ $\mathrm{H}_{2} \mathrm{O}$ under general anesthesia, ${ }^{27,28}$ peak airway pressure below $25 \mathrm{~cm} \mathrm{H}_{2} \mathrm{O}$ is unlikely to cause significant gastric insufflation. In previous reports, significant gastric insufflation was not observed during supraglottic ventilation, which is consistent with our results. ${ }^{17,18}$ However, there are some clinical situations where our device might induce gastric insufflations with high driving pressure, in any patient with an incompetent lower esophageal sphincter, such as after a cardiac arrest. ${ }^{29,30}$ Further clinical studies are needed to verify the safety of our device in these settings.

There are several limitations to this study. First, this study was not conducted in a real human, although the lung model and the intubation mannequin were adjusted to simulate adult clinical situations. Results from this study should be cautiously extrapolated to actual patient care until clinical studies can be conducted. Second, we only simulated an adult patient, and our study results may not apply to pediatric patients whose airway size and respiratory mechanics are quite different. Third, only 2 videolaryngoscopes were tested. Because the design features of individual scopes differ, all scopes should be evaluated before assuming results would be the same as ours. We believed that the efficacy of ventilation mainly depends on the distance from the tip of the ventilation tube to the vocal cords and the angulations of the nozzle as related to the tracheal axis.

In conclusion, the VLs-Vent can produce effective ventilation in the presence of normal respiratory system mechanics as long as the vocal cords are visualized. Because video-laryngoscopes provide a better view of vocal cords than direct laryngoscopes, this novel system can potentially improve ventilation/oxygenation during endotracheal intubation. Further clinical studies are needed to validate our observation, and to define specific design features and optimize its functionality.

\section{REFERENCES}

1. Benumof JL. Management of the difficult adult airway: with special emphasis on awake tracheal intubation. Anesthesiology 1991;75(6): 1087-1110.

2. Wong E, Ng YY. The difficult airway in the emergency department. Int J Emerg Med 2008;1(2):107-111.

3. Orebaugh SL. Difficult airway management in the emergency department. J Emerg Med 2002;22(1):31-48.

4. Taryle DA, Chandler JE, Good JT Jr, Potts DE, Sahn SA. Emergency room intubations: complications and survival. Chest 1979;75(5): 541-543.

5. Stewart RD, Paris PM, Winter PM, Pelton GH, Cannon GM. Field endotracheal intubation by paramedical personnel: success rates and complications. Chest 1984;85(3):341-345.

6. European Resuscitation Council. European Resuscitation Council Guidelines for Resuscitation 2005. Resuscitation 2005;67:S1-S190.

7. Maruyama K, Tsukamoto S, Ohno S, Kobayashi K, Nakagawa H, Kitamura A, Hayashida M. Effect of cardiopulmonary resuscitation on intubation using a Macintosh laryngoscope, the AirWay Scope, 


\section{Video-Laryngoscopes With a Ventilation Feature}

and the gum elastic bougie: a manikin study. Resuscitation 2010; 81(8):1014-1018.

8. Cheney FW, Posner KL, Lee LA, Caplan RA, Domino KB. Trends in anesthesia-related death and brain damage: a close claims analysis. Anesthesiology 2006;105(6):1081-1086.

9. Sirian R, Wills J. Physiology of apnoea and the benefits of preoxygenation. Contin Educ Anaesth Crit Care Pain 2009;9(4):105108.

10. Jaber S, Amraoui J, Lefrant JY, Arich C, Cohendy R, Landreau L, Calvet Y, Capdevila X, Mahamat A, Eledjiam JJ, et al. Clinical practice and risk factors for immediate complications of endotracheal intubation in the intensive care unit: a prospective, multi-center study. Crit Care Med 2006;34(9):2355-2361.

11. Healy DW, Maties O, Hovord D, Kheterpal S. A systematic review of the role of videolaryngoscopy in successful orotracheal intubation. BMC Anesthesiol 2012;12:32.

12. Sakles JC, Mosier J, Chiu S, Cosentino M, Kalin L. A comparison of the C-MAC video laryngoscope to the Macintosh direct laryngoscope for intubation in the emergency department. Ann Emerg Med 2012;60(6):739-748.

13. Niforopoulou P, Pantazopoulos I, Demestiha T, Koudouna E, Xanthos T. Video-laryngoscopes in the adult airway management: a topical review of the literature. Acta Anesthesiol Scand 2010;54(9): 1050-1061.

14. Serocki G, Bein B, Scholz J, Dörges V. Management of the predicted difficult airway: a comparison of conventional blade laryngoscopy with video-assisted blade laryngoscopy and the GlideScope. Eur J Anaesth 2010;27(1):24-30.

15. Kory P, Guevarra K, Mathew JP, Hegde A, Mayo PH. The impact of video laryngoscopy use during urgent endotracheal intubation in the critically ill. Anesth Analg 2013;117(1):144-149.

16. Cormack RS, Lehane J. Difficult tracheal intubation in obstetrics. Anaesthesia 1984;39(11):1105-1111.

17. Wei H. A new tracheal tube and methods to facilitate ventilation and placement in emergency airway management. Resuscitation 2006; 70(3):438-444.

18. Peng J, Ye J, Zhao Y, Liang J, Huang H, Wei H, Peng S. Supraglottic jet ventilation in difficult airway management. J Emerg Med 2012; 43(2):382-390.
19. Teoh WH, Saxena S, Shah MK, Sia AT. Comparison of three videolaryngoscopes: Pentax Airway Scope, C-MAC, GlideScope vs the Macintosh laryngoscope for tracheal intubation. Anaesthesia 2010; 65(11):1126-1132.

20. Ng SY, Ithnin E, Lim Y. Comparison of airway management during anaesthesia using the laryngeal mask airway CTrach and Glidescope. Anaesth Intensive Care 2007;35(5):736-742.

21. American Society of Anesthesiologists Task Force on Management of the Difficult Airway. Practice guidelines for management of the difficult airway: an updated report by the American Society of Anesthesiologists Task Force on Management of the Difficult Airway. Anesthesiology 2003;98(5):1269-1277.

22. Henderson JJ, Popat MT, Latto IP, Pearce AC, Difficult Airway Society. Difficult Airway Society guidelines for management of the unanticipated difficult intubation. Anaesthesia 2004;59(7):675-694.

23. Bourgain JL, Desruennes E, Fischler M, Ravussin P. Transtracheal high frequency jet ventilation for endoscopic airway surgery: a multicentre study. Br J Anaesth 2001;87(6):870-875.

24. Patel RG. Percutaneous transtracheal jet ventilation: a safe, quick, and temporary way to provide oxygenation and ventilation when conventional methods are unsuccessful. Chest 1999;116(6):1689-1694.

25. Jacobs HB, Smyth NP, Witorsch P. Transtracheal catheter ventilation: clinical experience in 36 patients. Chest 1974;65(1):36-40.

26. Rezaie-Majd A, Bigenzahn W, Denk DM, Burian M, Kornfehl J, Grasl M. Ch, Ihra G, Aloy A. Superimposed high-frequency jet ventilation (SHFJV) for endoscopic laryngotracheal surgery in more than 1500 patients. Br J Anaesth 2006;96(5):650-659.

27. Ho-Tai LM, Devitt JH, Noel AG, O’Donnell MP. Gas leak and gastric insufflation during controlled ventilation: face mask versus laryngeal mask airway. Can J Anaesth 1998;45(3):206-211.

28. Weiler N, Heinrichs W, Dick W. Assessment of pulmonary mechanics and gastric inflation pressure during mask ventilation. Prehosp Disaster Med 1995;10(2):101-105.

29. Bowman FP, Menegazzi JJ, Check BD, Duckett TM. Lower esophageal sphincter pressure during prolonged cardiac arrest and resuscitation. Ann Emerg Med 1995;26(2):216-219.

30. Lawes EG, Baskett PJ. Pulmonary aspiration during unsuccessful cardiopulmonary resuscitation. Intensive Care Med 1987;13(6): 379-382.

This article is approved for Continuing Respiratory Care Education credit. For information and to obtain your CRCE

(free to AARC members) visit

\section{Www.rcjournal.com}

